\title{
RESOLVENT ESTIMATES WITH MILD TRAPPING
}

\author{
JARED WUNSCH
}

\begin{abstract}
We discuss recent progress in understanding the effects of certain trapping geometries on cut-off resolvent estimates, and thus on the qualititative behavior of linear evolution equations. We focus on trapping that is unstable, so that strong resolvent estimates hold on the real axis, and large resonance-free regions can be shown to exist beyond it.
\end{abstract}

\section{INTRODUCTION}

Let $(X, g)$ be a Riemannian manifold isometric to $\mathbb{R}^{n}$ outside a compact set. Let $\Delta$ denote the (non-negative) Laplace-Beltrami operator

$$
\Delta=\frac{1}{\sqrt{g}} D_{i} g^{i j} \sqrt{g} D_{j}
$$

where $D_{i}=-\imath \partial_{x_{i}}$. The resolvent

$$
\left(\Delta-\lambda^{2}\right)^{-1}
$$

is a priori defined as a family of bounded operators $L^{2}(X) \rightarrow L^{2}(X)$, as $\lambda$ runs over the upper half-space $\operatorname{Im} \lambda>0$. When the manifold is precisely $\mathbb{R}^{n}$ we can write down the Schwartz kernel of the resolvent by Fourier methods; for instance in $\mathbb{R}^{3}$ the Schwartz kernel is

$$
\frac{e^{i \lambda|x-y|}}{4 \pi|x-y|} \text {. }
$$

We thus see explicitly that this kernel analytically continues to be an entire function of $\lambda \in \mathbb{C}$. It has the modest defect of exponential growth at infinity, which is remedied if we consider it as a map $L_{c}^{2}\left(\mathbb{R}^{3}\right) \rightarrow L_{\text {loc }}^{2}\left(\mathbb{R}^{3}\right)$. The same holds true on $\mathbb{R}^{n}$ for all odd $n$.

Putting geometry back into the picture, we may regard $X$ as a perturbation of Euclidean space, and an easy application of analytic Fredholm theory (see, e.g., 38 in a much more general setting) shows that in general, for $n$ odd, $\left(\Delta-\lambda^{2}\right)^{-1}$ continues as a meromorphic family of operators

Date: October 31, 2018.

The author is grateful to Dean Baskin, Hans Christianson, Emmanuel Schenck, András Vasy, and Maciej Zworski both for scientific discussions and for helpful comments on this manuscript. The author also thanks David Lannes and the scientific committee of the CNRS GDR "Analyse des Equations aux Dérivées Partielles" for the opportunity to present the talk on which this exposition is based. This work was partially supported by NSF grant DMS-1001463. 


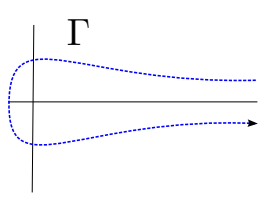

Figure 1. The contour of integration in (1).

$L_{c}^{2}(X) \rightarrow L_{\mathrm{loc}}^{2}(X)$, for all $\lambda \in \mathbb{C}$. The poles of this family of operators are known as the resonances of the Laplacian. We may equally well think of them as the poles of the cutoff resolvent family

$$
\chi\left(\Delta-\lambda^{2}\right) \chi: L^{2}(X) \rightarrow L^{2}(X)
$$

with $\chi \in \mathcal{C}_{c}^{\infty}(X)$, and we will adopt this point of view in what follows. (For a survey of some recent developments in the theory of resonances complementary to the discussion here, we refer the reader to [54].)

We note that while the new results described in this paper fit (more or less) in the geometric context described above, much of the history of the subject is closely tied to the problem where $X$ is replaced by an exterior domain of a smoothly bounded subset of $\mathbb{R}^{n}$ (the "obstacle problem") equipped with, say, Dirichlet boundary conditions. As the obstacle problem has considerable similarities to that considered here, we will cite results on the obstacle problem as motivation without further comment.

We now recall two different estimates in the theory of evolution equations that follow from an "appropriate" understanding of the resolvent continued to the real axis and beyond.

1.1. Energy decay for the wave equation. Let $U(t)=\sin t \sqrt{\Delta} / \sqrt{\Delta}$ be (part of the) wave propagator. Then we may write $U(t)$ as a contour integral around the spectrum of the Laplacian, which is in this situation simply continuous spectrum along the positive real axis. Thus, letting $\Gamma$ denote the contour shown in Figure 1.1, we have

$$
\begin{aligned}
\chi U(t) \chi & =\frac{1}{2 \pi \imath} \int_{\Gamma} \chi(\mu-\Delta)^{-1} \chi \frac{\sin t \sqrt{\mu}}{\sqrt{\mu}} d \mu \\
& =-\frac{1}{\pi \imath}\left(\int_{\mathbb{R}+\imath 0} \chi R(\lambda) \chi e^{\imath t \lambda} d \lambda+\int_{\mathbb{R}+\imath 0} \chi R(\lambda) \chi e^{-\imath t \lambda} d \lambda\right)
\end{aligned}
$$

Here we have made the change of variables $\mu=\lambda^{2}$ and pushed the contour within $\epsilon$ of the real axis for any $\epsilon>0$ (hence the contour is written $\mathbb{R}+\imath 0$ ).

Now suppose we are interested in the local large- $t$ asymptotics of a solution to the wave equation, i.e., of $\chi U(t) f$ for $f$ compactly supported, or equivalently to $\chi U(t) \chi g$ for any $g \in L^{2}(X)$; here $\chi$ is again a compactly supported cutoff function. For $t \gg 0$, can can always slide the contour of integration in the first integral on the last line of (1) upward to $\mathbb{R}+\imath \nu(\nu>0)$ and we get exponential decay, $O\left(e^{-\nu t}\right)$ for this term. The second integral, 


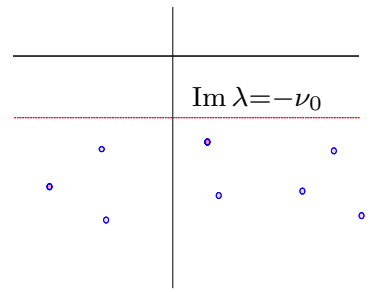

FiguRE 2. A resonance-free strip.

however, is trickier, as we would wish to slide this contour downward below the real axis, where we only know that the resolvent is meromorphic.

In order to deal with this second term, let us now suppose we have:

(1) $\chi\left(\Delta-(\mu+\imath \nu)^{2}\right)^{-1} \chi$ analytic for $-\nu_{0} \leq \nu \leq 0$.

(2) $\left\|\chi\left(\Delta-(\mu+\imath \nu)^{2}\right)^{-1} \chi\right\|_{L^{2}} \lesssim\langle\mu\rangle^{N}$ in this strip ${ }^{1}$

If these conditions hold, then we can shift the second contour, at the cost of some powers of $\mu$ or, equivalently, powers of $\Delta$ and for $K$ sufficiently large get the bound $O\left(e^{-\nu t}\right)$ for any $\nu<\nu_{0}$ for the smoothed wave operator $\chi\langle\Delta\rangle^{-K} U(t) \chi$. Thus we obtain exponential decay of local energy (potentially with derivative loss).

We further remark that in flat space, $N=-1$ in the second condition and we in fact have no derivative loss in the estimates: whenever $\left(D_{t}^{2}-\Delta\right) u=0$ and $u$ has compactly supported Cauchy data,

$$
\left\|\chi u_{t}\right\|^{2}+\|\chi \nabla u\|^{2} \leq C e^{-\nu t}\left(\left\|u_{t}(0)\right\|^{2}+\|\nabla u(0)\|^{2}\right)
$$

(where the constant may depend on the size of the support).

1.2. Local smoothing for the Schrödinger equation. We now consider a second application, which only involves the cut-off resolvent estimate on the real axis. This application is to the initial value problem for the timedependent Schrödinger equation:

$$
\left(\imath^{-1} \frac{\partial}{\partial t}+\Delta\right) u=0,\left.u\right|_{t=0}=u_{0} .
$$

The estimate on the cut-off resolvent that holds on the real axis in Euclidean space,

$$
\|\chi R(\mu) \chi\|_{L^{2}} \lesssim\langle\mu\rangle^{-1}
$$

implies the following property of $u$ :

$$
\int_{0}^{1}\|\chi u\|_{H^{1 / 2}}^{2} d t \lesssim\left\|u_{0}\right\|_{L^{2}}^{2}
$$

In other words, we have the following mapping property of the propagator:

$$
e^{-\imath t \Delta}: L^{2} \rightarrow L^{2}\left([0,1] ; H_{\text {loc }}^{1 / 2}(X)\right)
$$

\footnotetext{
${ }^{1}$ Here and henceforth, $f \lesssim g$ means $f \leq C g$ for some constant $C$.
} 
(see Constantin-Saut [13], Sjölin [37, Vega [49], Kato-Yajima [28, Yajima [51]). A proof that (3) gives this estimate proceeds via a standard " $T T^{*}$ " estimate which reduces to proving the corresponding estimate on the inhomogeneous equation:

$$
\left(\imath^{-1} \frac{\partial}{\partial t}+\Delta\right) v=\chi f,\left.u\right|_{t=0}=0 \Longrightarrow\|\chi u\|_{L_{t}^{2} H_{x}^{1 / 2}} \lesssim\|\chi f\|_{L_{t}^{2} H_{x}^{-1 / 2}}
$$

This inhomogeneous estimate, in turn, follows from Fourier transform and (3).

1.3. Semi-classical rescaling, and estimates in non-trapping geometries. Having established that cut-off resolvent estimates on and below the real axis are of key importance in the study of the wave and Schrödinger equations, we now pursue the question of when they hold in non-Euclidean geometries. To begin, we note that both the validity of the estimate (3) and also of the existence of a pole-free strip with polynomial resolvent bounds are high-frequency questions: they manifestly hold in any compact range of $\operatorname{Re} \lambda$, hence they hinge only on the asymptotics of the resolvent as $\operatorname{Re} \lambda \rightarrow+\infty$. Consequently, we now introduce a semi-classical rescaling of the problem: we set

$$
\lambda^{2}=\frac{z}{h^{2}}
$$

and study the operator family

$$
P_{h}(z) \equiv h^{2} \Delta-z
$$

and its inverse

$$
R_{h}(z) \equiv\left(h^{2} \Delta-z\right)^{-1} .
$$

We now note that the existence of a resonance free strip in $\lambda$ is equivalent to pole-free region in $z$ for $\chi R_{h}(z) \chi$ of form

$$
[1-\delta, 1+\delta]+i\left[-\nu_{0} h, 0\right]
$$

and the desired polynomial growth estimate in $\operatorname{Re} \lambda$ becomes a polynomial estimate in $h^{-1}$. Likewise the free resolvent estimate on the real axis (3) rescales to:

$$
\left\|\chi R_{h}(z) \chi\right\| \lesssim h^{-1}, \quad z \in[1-\delta, 1+\delta]
$$

In order to explain a broad setting in which these estimates generalize, we now introduce the notion of trapped set. Let $\mathrm{H}_{p}$ denote the Hamilton vector field of $p=\sigma_{h}(P)$, i.e., the generator of geodesic flow in the cosphere bundle. Then the trapped set $K$ is defined by

$$
\rho \in K \Longleftrightarrow \exp \left(t \mathrm{H}_{p}\right) \text { remains in a compact set for all } t \text {. }
$$

We say the metric is non-trapping if $K=\emptyset$.

The following is the classical result on estimates for non-trapping metrics: 
Proposition 1. If the metric is nontrapping then there is a resonance free region of the form

$$
[1-\delta, 1+\delta]+\imath\left[-\nu_{0} h|\log h|, 0\right]
$$

and the free resolvent estimate holds:

$$
\left\|\chi R_{h}(z) \chi\right\| \lesssim h^{-1}, z \in[1-\delta, 1+\delta] .
$$

Indeed, a somewhat stronger statement holds on the distribution of resonances: for $h$ small, the region $[1-\delta, 1+\delta]+\imath[-\nu h|\log h|, 0]$ contains only finitely many resonances, for any value of $\nu$.

These estimates have a lengthy history in both the obstacle case and the metric setting considered here. The deduction of exponential energy decay from the eventual smoothness of solutions to the wave equation with compactly supported data (a very weak form of Huygens' Principle) follows from work of Lax-Phillips [29] and Vainberg [46, 47]. (For further references on the propagation of singularities arguments leading to these estimates in the obstacle case, we refer the reader to [43]; in the metric case dealt with here, it simply follows from Hörmander's theorem on propagation of singularities for operators of real principal type [27].)

The estimate on the real axis is sharp: Ralston [34] showed (in the obstacle setting) that the estimate on the real axis can only hold if the trapped set is empty. Indeed, if there is stable trapping, e.g. a closed elliptic geodesic, then it is known that the resolvent grows on $\mathbb{R}$, with resonances approaching the real axis, as $\operatorname{Re} \lambda \rightarrow+\infty$ (see e.g., [39, 40, 42], 41]). The minimal size of the resonance free strip was established by Burq [8], who showed that there must always be a region of the form $\operatorname{Im} \lambda>-C e^{-\epsilon|\lambda|}$ free of poles of $\left(\Delta-\lambda^{2}\right)$ and consequently there is energy decay for the wave equation at the rate $\log (2+t)^{-k}$ if we accept loss of $k$ derivatives.

\section{Resolvent estimates in the PRESEnCE OF Mild tRAPPing}

2.1. Normally hyperbolic trapping. What, then, if there are trapped orbits, i.e., $K \neq \emptyset$, but the trapping is at least unstable? The classical example of Ikawa sheds considerable light on this problem: in the case of the exterior problem for two strictly convex obstacles, there is a unique trapped orbit consisting of the single orbit bouncing back and forth between them; this orbit is highly unstable, and Ikawa was able to show that in this case there is a strip $\operatorname{Im} z \geq-\nu_{0} h$ that is free of resonances, and indeed to derive asymptotics for the locations of the resonances. A refinement of Tang-Zworski [43] and Burq [5] shows that while the non-trapping estimate cannot hold on the real axis, we still have:

$$
R_{h}(\lambda) \lesssim \frac{|\log h|}{h}, \quad \lambda \in[1-\delta, 1+\delta] .
$$

In other words, we lose only a factor of $|\log h|$ relative to the non-trapping estimate. This implies, among other things, that the local smoothing estimate 
for the Schrödinger propagator only barely fails: for any $\epsilon>0$,

$$
e^{-\imath t \Delta}: L^{2} \rightarrow L^{2}\left([0,1] ; H_{\mathrm{loc}}^{1 / 2-\epsilon}\right) .
$$

In the boundary-less case, the analogous situation in which we have a single unstable hyperbolic orbit (e.g., the unique closed geodesic on the surface of revolution shown in Figure 4 below) was analyzed by Christianson [12. More generally, the author and Zworski [50] have recently studied the situation of normally hyperbolic trapping, in which there is a whole manifold of unstable trapped orbits. We now describe these results.

One motivation for considering this geometric set-up comes from the Kerr black hole. This is a family of Lorentzian metrics which solve the Einstein equations and describe rotating black holes. The trapped set of null-geodesics (known as the "photon sphere" in the special, spherically symmetric, case of the Schwarzschild metric) has the dynamical structure described below, and thus the results of [50] address the obstructions to decay posed by the trapped rays. Considerable geometric and analytic difficulties are present in the study of wave decay on Kerr backgrounds that are not addressed by [50] however; see, e.g. [44, [45] [19], [14, [1, 23, 24] for results along these lines and for further references. In the related setting of Kerr-de-Sitter black hole metric: ${ }^{2}$ and their perturbations, some of the obstructions to decay posed by the asymptotically Euclidean end of the de Sitter space are not present. In the case of slowly-rotating ${ }^{3}$ Kerr-de-Sitter metrics, Dyatlov [21, 20] used the results from [50] presented below to show that wave equation solutions decay exponentially to a constant value; Vasy [48] was able to extend such decay estimates to a wide class of perturbations of Kerr-de-Sitter space, again using the results of [50] to cope with the trapped set.

The dynamical hypotheses employed in [50] are as follows. For simplicity of exposition here we stick to the geometric set-up described above, where we consider the operator $h^{2} \Delta$ on the manifold $(X, g)$ with $g$ Euclidean outside a compact set. Let $\varphi^{t}$ denote the corresponding geodesic flow. Let $r$ denote the distance function to a fixed point in $X$ and locally define the backward/forward trapped sets by:

$$
\Gamma_{ \pm}=\left\{\rho: \lim _{t \rightarrow \mp \infty} r\left(\varphi^{t}(\rho)\right) \neq \infty\right\} .
$$

The the trapped set is simply

$$
K=\Gamma_{+} \cap \Gamma_{-} .
$$

We make dynamical assumptions as follows:

\footnotetext{
${ }^{2}$ These metrics describe rotating black holes in a universe with positive cosmological constant.

${ }^{3}$ Slowly rotating means that the angular momentum parameter in the metric, usually denoted $a$, is taken to be small.
} 
(1) $\Gamma_{ \pm}$are codimension-one smooth manifolds intersecting transversely at $K$. (It is not difficult to verify that $\Gamma_{ \pm}$must then be coisotropic and $K$ symplectic.)

(2) The flow is hyperbolic in the normal directions to $K$ within the energy surface: letting the superscript 1 denote the intersection of the set in question with the unit cosphere bundle (e.g., $K^{1}=K \cap S^{*} X$ ) then there exist subbundles $E^{ \pm}$of $T_{K^{1}} \Gamma_{ \pm}^{1}$ (the "unstable/stable subspaces") such that

$$
T_{K^{1}} \Gamma_{ \pm}^{1}=T K^{1} \oplus E^{ \pm}
$$

where

$$
d \varphi^{t}: E^{ \pm} \rightarrow E^{ \pm}
$$

and there exists $\theta>0$ such that on $K^{1}$,

$$
\left\|d \varphi^{t}(v)\right\| \leq C e^{-\theta|t|}\|v\| \text { for all } v \in E^{\mp}, \pm t \geq 0 .
$$

These assumptions can be verified directly (see [50]) for the trapped set of a slowly rotating Kerr black hole ${ }^{4}$ (i.e. when the angular momentum parameter $a$ is small - see [50] for the explicit form of the metric) but they are not stable under perturbations, hence do not obviously apply to perturbations of Kerr metrics. However, the bicharacteristic flow for the Kerr metric in fact satisfies a more stringent (and well-studied) hypothesis that is stable under perturbation, and that implies the dynamical hypotheses above. In particular, the standard dynamical notion of $r$-normal hyperbolicity implies items (1) and (2), and is stable under perturbations, modulo possible loss of derivatives. This hypothesis says, roughly speaking, that along $K$ the tangent space of the normal bundle to $K$ splits into subbundles $E^{ \pm}$ which are exponentially expanded/contracted by the flow, while the expansion/contraction of directions along $T K$ is much milder by comparison (e.g., polynomial in the case of the Kerr metric). It is then a profound theorem of Fenichel [22] and Hirsch-Pugh-Shub [26] that there exist stable/unstable manifolds $\Gamma_{ \pm}$tangent to $E^{ \pm}$and satisfying the dynamical hypotheses above, and moreover that these stronger hypotheses are structurally stable. ${ }^{5}$

Our main result on the resolvent for normally hyperbolic trapping is as follows:

Theorem 2. (Wunsch-Zworski [50]) Under the normal hyperbolicity hypotheses described above, there exist $\delta, \nu_{0}>0$ such that $\left(h^{2} \Delta-z\right)^{-1}$ is meromorphic in the region

$$
[1-\delta, 1+\delta]+\imath\left[-\nu_{0} h, 0\right]
$$

\footnotetext{
${ }^{4}$ See also 48 for analogous discussion of the Kerr-de-Sitter metric.

${ }^{5}$ Albeit in general, perturbing results in loss of differentiability of the manifolds $\Gamma_{ \pm}$and $K$; however for any desired finite degree of differentiability, shrinking the perturbation preserves that degree of smoothness, which suffices for our purposes.
} 
and the following polynomial resolvent estimates on the hold: on the real axis, we have

$$
\left\|\chi R_{h}(\lambda) \chi\right\| \lesssim \frac{|\log h|}{h}, \lambda \in[1-\delta, 1+\delta],
$$

while below the axis there exists $k>0$ such that

$$
\left\|\chi R_{h}(z) \chi\right\| \lesssim h^{-k}, z \in[1-\delta, 1+\delta]+\imath\left[-\nu_{0} h, 0\right] .
$$

In the case when the operator has real analytic coefficients a more precise resonance free region was obtained by Gérard-Sjöstrand [25], albeit without the polynomial bound on the resolvent that makes contour deformation (with loss of derivatives) feasible to obtain exponential energy decay for solutions to the wave equation. A similar estimate on the optimal width of the resonance free strip in the setting considered here, and with much reduced smoothness assumptions on the stable/unstable manifolds $\Gamma_{ \pm}$, has been recently announced by Nonnenmacher-Zworski [52].

Estimates like Theorem 2 have also been proved in the case when $K$ is fractal rather than a smooth manifold: provided the set is sufficiently filamentary, in a manner measured by a topological pressure condition, an estimate of the same form was previously proved by Nonnenmacher-Zworski [32]; in [33] these authors also obtain more precise resolvent bounds below the real axis. (See also Christianson [11] and Datchev [17] for applications to Schrödinger local smoothing in the presence of various assumptions on the ends of $X$.)

2.2. Degenerate hyperbolic trapping. Since stable trapping, for instance by elliptic closed geodesics, generates exponentially growing resolvent estimates, while unstable trapping with hyperbolic dynamics leads only to a $\operatorname{loss}$ of $|\log h|$ on the real axis, the reader might wonder whether there are situations in which a nontrivial polynomial loss occurs. This phenomenon was demonstrated by the author and Hans Christianson [10] in the following situation: We consider the manifold $X=\mathbb{R}_{x} \times \mathbb{R}_{\theta} / 2 \pi \mathbb{Z}$, equipped with a metric of the form

$$
d s^{2}=d x^{2}+\left(1+x^{2 m}\right)^{1 / m} d \theta^{2},
$$

Here the trapped set is again the closed orbit where $x=0$. If $m=1$, this is again the classic case of a single trapped hyperbolic orbit, but if $m$ is an integer greater than 1 the hyperbolicity degenerates with the stable and unstable manifold no longer intersecting transversely. Christianson and the author showed the following:

Theorem 3. (Christianson-Wunsch [10]) For $m$ an integer at least 2,

$$
\left\|\chi R_{h}(\lambda) \chi\right\| \lesssim h^{-2 m /(m+1)}, \quad \lambda \in[1-\delta, 1+\delta]
$$

and this estimate is sharp. 
Consequently, the optimal local smoothing estimate in this situation entails a nontrivial loss of derivatives:

$$
e^{-\imath t \Delta}: L^{2} \rightarrow L^{2}\left([0,1] ; H_{\mathrm{loc}}^{1 /(m+1)}\right),
$$

and we may obtain a polynomial rather than exponential rate of energy decay for the wave equation.

2.3. Trapping by cone points. While metrics with trapped rays always have losses in resolvent estimates on the real axis and in Schrödinger local smoothing (see Doi [18] and Burq-Bony-Ramond [4] as well as Ralston [34] in the obstacle case), we now present a situation involving wave propagation on singular manifolds in which a weaker form of "diffractive" trapping results in (almost) no loss whatsoever. This is the setting of manifolds with cone points.

In joint work with Dean Baskin, the author considers a manifold $X$ of dimension $n$ with conic singularities that is isometric to $\mathbb{R}^{n}$ outside a compact set. Here a manifold with conic singularities means a manifold $X$ with compact boundary $Y=\partial X$ equipped with a metric that can be brought to the form

$$
g=d x^{2}+x^{2} h
$$

in a neighborhood of $Y$, with $h$ a smooth tensor restricting to give a metric on $Y$. The metric thus degenerates at the boundary, and each boundary component becomes a cone point.

In considering trapping, we must now distinguish two kinds of geodesic on $X$. Following Melrose-Wunsch [31, we let a diffractive geodesic denote a geodesic that can enter and leave a cone point (i.e., a single boundary component) along any pair of geodesics. By contrast, a geometric geodesic is one that is restricted to enter and leave a single cone point along a pair of geodesics that are connected by a geodesic of length $\pi$ inside $Y$ (with respect to the metric $\left.h\right|_{Y}$ ). In [31, the geometric geodesics are shown to be exact those that are (locally) approximable by ordinary geodesics in $X^{\circ}$.

We impose geometric hypotheses that

(1) The flow along geometric geodesics is non-trapping.

(2) No three cone points are collinear along a geometric geodesic.

(3) No two cone points are conjugate to one another.

(The last condition can be most easily interpreted as saying that the geodesic flowouts from two different cone points intersect transversely.)

Then the following estimate on the resolvent holds:

Theorem 4. (Baskin-Wunsch [3]) For $\chi \in \mathcal{C}_{c}^{\infty}(X)$, there exist $\delta, \nu_{0}>0$ such that the cut-off resolvent

$$
\chi\left(h^{2} \Delta-z\right)^{-1} \chi
$$

can be analytically continued from $\operatorname{Im} z>0$ to the region

$$
z \in[1-\delta, 1+\delta]+\imath\left[-\nu_{0} h|\log h|, 0\right]
$$


and for some $C, T>0$ enjoys the estimate

$$
\left\|\chi\left(h^{2} \Delta-z\right)^{-1} \chi\right\|_{L^{2} \rightarrow L^{2}} \leq \frac{C}{h} e^{T|\operatorname{Im} z| / h}
$$

in this region.

This implies that the trapping of waves induced by diffraction among cone points has only a very weak effect on the decay of solutions to the wave equation on such a manifold: in particular, solutions to the wave equation on $\mathbb{R} \times X$ enjoy exponential local energy decay. Likewise, the resolvent estimate on the real axis (which is the same as would be obtained with no trapped rays) implies that the Schrödinger propagator $e^{-\imath t \Delta}$ enjoys the local smoothing estimate with no loss, mapping $L^{2} \rightarrow L^{2}\left([0,1]_{t} ; H_{\mathrm{loc}}^{1 / 2}(X)\right)$.

The theorem is proved by first establishing a result on weak escape of singularities for solutions to the wave equation. We recall that in odd dimensional Euclidean space, Huygens' Principle tells us that any solution to the wave equation with compactly supported Cauchy data is eventually zero in any fixed compact set $F$; in a non-trapping perturbation of Euclidean space (of any dimension), the solution does not in general vanish after long time but instead, by propagation of singularities, is eventually in $\mathcal{C}^{\infty}(F)$. In the conic situation, by contrast, we expect that singularities may persist forever in a compact set, diffracting back and forth among the cone points, but it turns out that they become milder and milder as time passes:

Theorem 5. (Baskin-Wunsch [3]) For any $r$ there exists $T_{r}$ such that for $|t|>T_{r}$, for all $s$,

$$
\chi \cos t \sqrt{\Delta} \chi: \mathcal{D}_{s} \rightarrow \mathcal{D}_{s+r} .
$$

(Here $\mathcal{D}_{s}$ is the domain of $\Delta^{s / 2}$ and agrees with $H^{s}$ away from the cone points.) One can then deduce Theorem 4 from Theorem 5 by adapting an argument of Vainberg [47] that employs the Fourier transform of the outgoing singular parts of the solution as an initial parametrix for the resolvent, solving away the resulting errors by using the free wave operator (for which the resolvent estimate assuredly holds).

One especially interesting and elementary setting in which our result on resonance-free regions applies is that of exterior domains to polygons in $\mathbb{R}^{2}$. We can double such domains by gluing together two copies along their boundary to obtain a manifold with cone points; solving the wave equation with Dirichlet/Neumann boundary conditions is equivalent to working with odd/even solutions on the doubled manifold. Provided the domain is nontrapping in the sense that billiard trajectories missing the cone points escape to infinity, we can thus show that there is a logarithmic region free of resonances for the obstacle problem with either boundary condition 6

\footnotetext{
${ }^{6}$ There is some mild technical complication from the fact that the manifold has two Euclidean ends, rather than just one as in our hypotheses, but the same strategy of proof applies.
} 
We remark that the size of the resonance free region in Theorem 5 is probably sharp: in the related situation of the exterior problem in $\mathbb{R}^{2}$ outside two strictly convex analytic obstacles, one having a corner, Burq [7] has shown that resonance poles for $\left(\Delta-\lambda^{2}\right)^{-1}$ lie (asymptotically) along curves of the form $\operatorname{Im} \lambda=C|\operatorname{Re} \lambda|+D$. The poles are thus at distance $O(h|\log h|)$ from the real axis after semiclassical rescaling. (We also refer the reader to Zworski [53] where analogous logarithmic strings of poles are shown to arise from finite order singularities of a one-dimensional potential, thus substantiating heuristics from Regge [36].) Consequently, the only loss in this setting of "diffractive trapping" relative to the non-trapping case is that we expect that some region of the form $z \in[1-\delta, 1+\delta]+\imath\left[-\nu_{0} h|\log h|, 0\right]$ probably contains infinitely many resonances in the former case, while this is not so in the latter.

\section{From Resolvent estimates to Damped waves}

We now turn to a problem related to, but crucially distinct from, that of resolvent estimates and decay for solutions to the wave equation: we now consider the damped wave equation on a compact, connected Riemannian manifold $X$. We fix a nonnegative "damping function" $a(x) \in \mathcal{C}^{\infty}$ and consider the initial value problem: ${ }^{7}$

$$
\left\{\begin{array}{l}
\left(\partial_{t}^{2}+\Delta_{g}+a(x) \partial_{t}\right) u(x, t)=0 \\
u(x, 0)=u_{0} \in H^{1}(X), \quad \partial_{t} u(x, 0)=u_{1} \in H^{0}(X)
\end{array}\right.
$$

It is well known [2, 30] that if $a>0$ somewhere, then any solution tends to zero as $t \rightarrow+\infty$. Moreover Lebeau has shown (see [30]) that the energy of the waves,

$$
E(u, t)=\frac{1}{2} \int_{X}\left(\left|\partial_{t} u\right|^{2}+|\nabla u|^{2}\right) d x
$$

decays at a logarithmic rate provided the data lie in a space with higher regularity than the energy space; this is of course analogous to the results discussed above for energy decay of solutions to the wave equation on noncompact manifolds in the presence of trapping.

To understand decay rates for the damped wave equation in further detail, we make a semiclassical reduction: Fourier transforming, it turns out that we can replace the damped wave operator by an operator of the form

$$
h^{2} \Delta+\imath h \sqrt{z} a-z
$$

exponential energy decay then follows from the existence of a pole-free region of the form

$$
z \in[1-\delta, 1+\delta]+\imath\left[-\nu_{0} h, 0\right]
$$

together with polynomial bounds for the inverse of this non-self-adjoint operator; smaller regions of analyticity yield sub-exponential decay rates. In

\footnotetext{
${ }^{7}$ Recall that the Laplacian employed here is the positive operator $\Delta=d^{*} d$.
} 
the results discussed here, the $z$ dependence of the middle term in (6) will be treatable as a perturbation in the operator above, and we study the family

$$
\left(h^{2} \Delta+\imath h a-z\right)^{-1}
$$

which is at least formally quite similar to the resolvent studied in previous sections.

Now in analogy with the trapped set in noncompact problems, we introduce the undamped set

$$
\mathcal{N}=\left\{\rho \in S^{*} X: \forall t \in \mathbb{R}, a \circ e^{t \mathrm{H}_{p}}(\rho)=0\right\}
$$

consisting of points that do not encounter the interior of the damping region under the forward- or backward-bicharacteristic flow. If $\mathcal{N}=\emptyset$ we are said to be in the situation of geometric control. Classic results of Rauch-Taylor [35], Bardos-Lebeau-Rauch [2] and Lebeau [30] show that in the setting of geometric control, we do have a pole-free region of the form (7) and exponential decay of energy. By contrast, if $\mathcal{N} \neq \emptyset$, it is known that exponential decay cannot hold without derivative losses. The situation is thus closely analogous to that described above for resolvent estimates, leading one to wonder if there is a link between the resolvent estimates for a noncompact manifold $\widetilde{X}$ with trapped set $K$ and estimates for (8) on a compact manifold $X$ with undamped set $\mathcal{N} \equiv K$, as long as the two manifolds are isometric in a neighborhood of the undamped/trapped set. In joint work with Hans Christianson, Emmanuel Schenck, and András Vasy, the author has proved that this is so: provided one has polynomial resolvent bounds on the real axis, one can "glue" these bounds to obtain the exact same estimate for the damped resolvent family (8). While our motivation comes from gluing on non-compact ends, the following theorem is in fact phrased in terms of the resolvent for the problem with "complex absorbing potential" "a replacing the damping coefficient $\imath h a$; such a potential has the effect of annihilating semiclassical wavefront set along (forward) bicharacteristics passing through it. Estimates with non-compact ends and with complex absorbing potential are known to be equivalent in a wide variety of geometric settings by a recent gluing theorem of Datchev-Vasy [16]. Phrasing the resolvent estimate in this way allows us to stay within the setting of compact manifolds, however:

Theorem 6. (Christianson-Schenck-Vasy-Wunsch [9]) Assume that for some $\delta \in(0,1)$ fixed and $k \in \mathbb{Z}$, there is a function $1 \leq \alpha(h)=O\left(h^{-k}\right)$ such that

$$
\left\|\left(h^{2} \Delta_{g}+\imath a-z\right)^{-1}\right\|_{L^{2} \rightarrow L^{2}} \leq \frac{\alpha(h)}{h},
$$

for $z \in[1-\delta, 1+\delta]$. Then there exist $C, \nu_{0}>0$ such that

$$
\left\|\left(h^{2} \Delta_{g}+\imath h a-z\right)^{-1}\right\|_{L^{2} \rightarrow L^{2}} \leq C \frac{\alpha(h)}{h},
$$

for $z \in[1-\delta, 1+\delta]+\imath\left[-\nu_{0} h / \alpha(h), 0\right]$. 


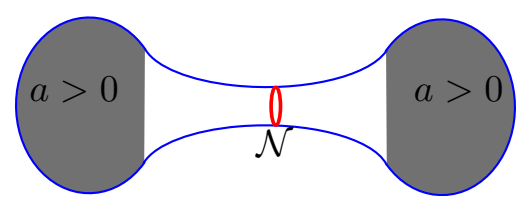

Figure 3. The compact manifold $X$ with damping.

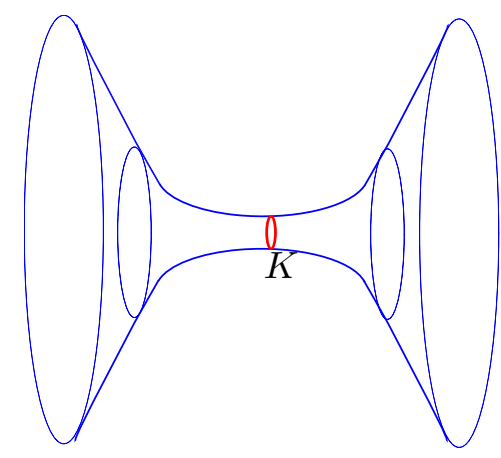

Figure 4. The noncompact manifold $\widetilde{X}$.

An essential new ingredient in the proof is a recently obtained estimate of Datchev-Vasy [15] which improves the microlocal resolvent estimates available at points which are backwards- or forwards-trapped relative to those which are trapped along both directions of the flow.

Theorem 6 has applications in all the examples discussed above (normally hyperbolic trapping, fractal hyperbolic trapping, degenerate hyperbolic trapping) giving various subexponential decay rates for solutions to the damped wave equation in these settings; we refer the reader to [9] for further details, including the explicit decay rates thus obtained. It is at least sometimes sharp, e.g. in the case of a surface of rotation with $\mathcal{N}$ given by an single undamped hyperbolic orbit, according to work of Burq-Christianson [6].

\section{REFERENCES}

[1] Lars Andersson and Pieter Blue. Hidden symmetries and decay for the wave equation on the Kerr spacetime, 2009.

[2] Claude Bardos, Gilles Lebeau, and Jeffrey Rauch. Sharp sufficient conditions for the observation, control, and stabilization of waves from the boundary. SIAM J. Control Optim., 30(5):1024-1065, 1992.

[3] D. Baskin and J. Wunsch. Resolvent estimates and local decay of waves on conic manifolds. In preparation.

[4] Jean-François Bony, Nicolas Burq, and Thierry Ramond. Minoration de la résolvante dans le cas captif. C. R. Math. Acad. Sci. Paris, 348(23-24):1279-1282, 2010.

[5] N. Burq. Smoothing effect for Schrödinger boundary value problems. Duke Math. J., 123(2):403-427, 2004.

[6] N. Burq and H. Christianson. Imperfect geometric control and overdamping for the damped wave equation. In preparation. 
[7] Nicolas Burq. Pôles de diffusion engendrés par un coin. Astérisque, (242):ii+122, 1997.

[8] Nicolas Burq. Décroissance de l'énergie locale de l'équation des ondes pour le problème extérieur et absence de résonance au voisinage du réel. Acta Math., 180(1):1-29, 1998.

[9] H. Christianson, E. Schenck, A. Vasy, and J. Wunsch. From resolvent estimates to damped waves. Preprint, 2012.

[10] H. Christianson and J. Wunsch. Local smoothing for the Schrödinger equation with a prescribed loss. Amer. J. Math., to appear.

[11] Hans Christianson. Cutoff resolvent estimates and the semilinear Schrödinger equation. Proc. Amer. Math. Soc., 136(10):3513-3520, 2008.

[12] Hans Christianson. Dispersive estimates for manifolds with one trapped orbit. Comm. Partial Differential Equations, 33(7-9):1147-1174, 2008.

[13] Peter Constantin and Jean-Claude Saut. Effets régularisants locaux pour des équations dispersives générales. C. R. Acad. Sci. Paris Sér. I Math., 304(14):407410, 1987.

[14] Mihalis Dafermos and Igor Rodnianski. Decay for solutions of the wave equation on kerr exterior spacetimes i-ii: The cases $|a| \ll m$ or axisymmetry, 2010.

[15] K. Datchev and A. Vasy. Semiclassical resolvent estimates at trapped sets. Preprint, 2012.

[16] K. Datchev and A. Vasy. Gluing semiclassical resolvent estimates via propagation of singularities. IMRN, to appear, Preprint, 2011.

[17] Kiril Datchev. Local smoothing for scattering manifolds with hyperbolic trapped sets. Comm. Math. Phys., 286(3):837-850, 2009.

[18] Shin-ichi Doi. Smoothing effects of Schrödinger evolution groups on Riemannian manifolds. Duke Math. J., 82(3):679-706, 1996.

[19] Roland Donninger, Wilhelm Schlag, and Avy Soffer. On pointwise decay of linear waves on a Schwarzschild black hole background. Comm. Math. Phys., 309(1):51-86, 2012.

[20] Semyon Dyatlov. Exponential energy decay for Kerr-de Sitter black holes beyond event horizons. Math. Res. Lett., 18(5):1023-1035, 2011.

[21] Semyon Dyatlov. Quasi-normal modes and exponential energy decay for the Kerr-de Sitter black hole. Comm. Math. Phys., 306(1):119-163, 2011.

[22] Neil Fenichel. Persistence and smoothness of invariant manifolds for flows. Indiana Univ. Math. J., 21:193-226, 1971/1972.

[23] F. Finster, N. Kamran, J. Smoller, and S.-T. Yau. Decay of solutions of the wave equation in the Kerr geometry. Comm. Math. Phys., 264(2):465-503, 2006.

[24] F. Finster, N. Kamran, J. Smoller, and S.-T. Yau. Erratum: "Decay of solutions of the wave equation in the Kerr geometry" [Comm. Math. Phys. 264 (2006), no. 2, 465-503;]. Comm. Math. Phys., 280(2):563-573, 2008.

[25] C. Gérard and J. Sjöstrand. Resonances en limite semiclassique et exposants de Lyapunov. Comm. Math. Phys., 116(2):193-213, 1988.

[26] M. W. Hirsch, C. C. Pugh, and M. Shub. Invariant manifolds. Springer-Verlag, Berlin, 1977. Lecture Notes in Mathematics, Vol. 583.

[27] L. Hörmander. On the existence and the regularity of solutions of linear pseudodifferential equations. Enseignement Math. (2), 17:99-163, 1971.

[28] Tosio Kato and Kenji Yajima. Some examples of smooth operators and the associated smoothing effect. Rev. Math. Phys., 1(4):481-496, 1989.

[29] P.D. Lax and R.S. Phillips. Scattering Theory. Academic Press, New York, 1967. Revised edition, 1989.

[30] G. Lebeau. Équation des ondes amorties. In Algebraic and geometric methods in mathematical physics (Kaciveli, 1993), pages 73-109. Kluwer Acad. Publ., Dordrecht, 1996.

[31] Richard Melrose and Jared Wunsch. Propagation of singularities for the wave equation on conic manifolds. Invent. Math., 156(2):235-299, 2004. 
[32] Stéphane Nonnenmacher and Maciej Zworski. Quantum decay rates in chaotic scattering. Acta Math., 203(2):149-233, 2009.

[33] Stéphane Nonnenmacher and Maciej Zworski. Semiclassical resolvent estimates in chaotic scattering. Appl. Math. Res. Express. AMRX, (1):74-86, 2009.

[34] James V. Ralston. Solutions of the wave equation with localized energy. Comm. Pure Appl. Math., 22:807-823, 1969.

[35] Jeffrey Rauch and Michael Taylor. Decaying states of perturbed wave equations. $J$. Math. Anal. Appl., 54(1):279-285, 1976.

[36] T. Regge. Analytic properties of the scattering matrix. Nuovo Cimento (10), 8:671679, 1958.

[37] Per Sjölin. Regularity of solutions to the Schrödinger equation. Duke Math. J., 55(3):699-715, 1987.

[38] J. Sjöstrand and M. Zworski. Complex scaling and the distribution of scattering poles. J. Amer. Math. Soc., 4:729-769, 1991.

[39] P. Stefanov and G. Vodev. Distribution of resonances for the Neumann problem in linear elasticity outside a strictly convex body. Duke Math. J., 78(3):677-714, 1995.

[40] P. Stefanov and G. Vodev. Neumann resonances in linear elasticity for an arbitrary body. Comm. Math. Phys., 176(3):645-659, 1996.

[41] Plamen Stefanov. Quasimodes and resonances: sharp lower bounds. Duke Math. J., 99(1):75-92, 1999.

[42] Siu-Hung Tang and Maciej Zworski. From quasimodes to resonances. Math. Res. Lett., 5(3):261-272, 1998.

[43] Siu-Hung Tang and Maciej Zworski. Resonance expansions of scattered waves. Comm. Pure Appl. Math., 53(10):1305-1334, 2000.

[44] Daniel Tataru. Local decay of waves on asymptotically flat stationary space-times. arXiv:0910.5290.

[45] Daniel Tataru and Mihai Tohaneanu. A local energy estimate on Kerr black hole backgrounds. Int. Math. Res. Not. IMRN, (2):248-292, 2011.

[46] B. R. Vaŭnberg. Exterior elliptic problems that depend polynomially on the spectral parameter, and the asymptotic behavior for large values of the time of the solutions of nonstationary problems. Mat. Sb. (N.S.), 92(134):224-241, 343, 1973.

[47] B. R. Vaŭnberg. Asymptotic methods in equations of mathematical physics. Gordon \& Breach Science Publishers, New York, 1989. Translated from the Russian by E. Primrose.

[48] A. Vasy. Microlocal analysis of asymptotically hyperbolic and kerr-de sitter spaces. Preprint, 2010.

[49] Luis Vega. Schrödinger equations: pointwise convergence to the initial data. Proc. Amer. Math. Soc., 102(4):874-878, 1988.

[50] Jared Wunsch and Maciej Zworski. Resolvent estimates for normally hyperbolic trapped sets. Ann. Henri Poincaré, 12(7):1349-1385, 2011.

[51] Kenji Yajima. On smoothing property of Schrödinger propagators. In Functionalanalytic methods for partial differential equations (Tokyo, 1989), volume 1450 of Lecture Notes in Math., pages 20-35. Springer, Berlin, 1990.

[52] M. Zworski. Personal communication.

[53] M. Zworski. Distribution of poles for scattering on the real line. J. Funct. Anal., 73:277-296, 1987.

[54] Maciej Zworski. Resonances in physics and geometry. Notices Amer. Math. Soc., 46(3):319-328, 1999. 